\title{
Reflexões sobre os efeitos da pandemia na aprendizagem digital
}

\author{
Reflection on the effects of the pandemic in the digital learning
}

\author{
Ana Nobre \\ Professor Auxiliar \\ Universidade Aberta - Uab \\ LE@D-Laboratório de Ensino a Distância \\ Lisboa - Portugal \\ ana.nobre@uab.pt \\ Ana Mouraz \\ Professor Auxiliar \\ Universidade Aberta - Uab \\ CIIE - Centro de Investigação e Intervenção Educativas \\ Lisboa - Portugal \\ ana.lopes@uab.pt
}

Resumo: No início da terceira década do século XXI, mudanças significativas estão a ocorrer nas Instituições de Ensino Superior (IES). Se a necessidade de oferecer educação superior a mais estudantes, e mais diversificados, vinha constituindo um desafio, imposto pela globalização e pela economia do conhecimento, a pandemia obrigou as IES a reequacionar a sua oferta digital. Embora o Ensino Superior use extensivamente o e-learning, o ensino on-line, o ensino a distância, ou alguma outra forma de educação tecnológica, importa refletir sobre os efeitos que tal uso trará à reconfiguração da aprendizagem digital, mesmo num período pós pandemia. É esse propósito que estrutura este texto. Parte-se da literatura recente sobre a aprendizagem digital e seus contornos para refletir sobre as possibilidades e dificuldades que a pandemia lhe trouxe.

Palavras-chave: Aprendizagem digital. Pandemia-COVID-19. Ensino superior. Globalização. Tecnologia digital.

Abstract: In the first beginning of the third decade of 21 century, a huge change is occurring in Higher Education. The need to offer higher education to more students, and more diversified, had been a challenge to Higher Education Institutions (HEIs), imposed by globalization and the knowledge economy. However, the pandemic forced HEIs to rebalance their digital offer. Although e-learning, online education, distance learning, or some other form of technological education have been used extensively in HE, it is important to reflect on the effects that such use will bring to the reconfiguration of digital learning, even in a postpandemic period. Such is the purpose that frame this text.

It starts from the recent literature on digital learning and its features to reflect on the possibilities and difficulties that the pandemic has brought to the concept and its practices.

Keywords: Digital learning. COVID-19 Pandemic. Higher Education. Globalization. Digital Technology. 


\section{Introdução}

A maioria das instituições do ensino superior direcionaram a sua oferta para um contexto online durante a pandemia global devido ao COVID-19. Se o conceito de aprendizagem digital vinha, paulatinamente, ganhando força e ocupando espaço nas preocupações e nas práticas das Instituições de Ensino Superior, a pandemia tornou-o uma emergência na razão direta do confinamento a que as Instituições educativas foram obrigadas. De repente toda a aprendizagem passou a ser digital.

O presente texto visa refletir sobre o conceito de aprendizagem digital, sobre as práticas que dão substância às suas caraterísticas para depois refletir, impressivamente, sobre os possíveis efeitos da pandemia na configuração da aprendizagem digital no Ensino Superior.

Trata-se de fazer um inventário impressivo desses efeitos, realizado a partir da literatura recente e da reflexão sobre as possibilidades e dificuldades que a pandemia lhe trouxe.

O texto organiza-se em torno de três objetivos que norteiam as três partes que o compõem, a saber: contextualizar a emergência do conceito de aprendizagem digital; revisitar as suas características no Ensino Superior; e refletir sobre os possíveis efeitos da pandemia na configuração da aprendizagem digital neste nível de ensino.

\section{Contextualização da emergência da aprendizagem digital}

A aprendizagem digital é o resultado da confluência dos desafios económicos e sociais da globalização reconfigurados pela tecnologia.

A globalização combina mudanças económicas, culturais e educacionais no mercado competitivo global (Englund, Olofsson, e Price, 2017). Em virtude desse caráter trouxe desafios sociais que se tornaram transversalmente relevantes a todos os níveis da vida em sociedade e em todos os setores de atividade, incluindo, necessariamente a educação.

Os padrões de qualidade que são exigidos por uma sociedade cada vez mais tecnológica e diversificada, local e globalmente (Lodge e Bonsanquet, 2014) tornam-se um mandato para as prioridades dos sistemas educativos. Entre as caraterísticas que a globalização aportou a essa reconfiguração estão a flexibilidade e a inovação.

Esses conceitos passaram a integrar o discurso das políticas educacionais em resposta à necessidade de uma maior articulação entre o subsistema educativo e o empresarial (Sin e Amaral,2017). Tais conceitos entraram também no discurso dos estudantes que procuram o ensino superior (Sin Tavares e Amaral,2016) e, enquanto critério de qualidade são inequivocamente 
essenciais para dar resposta aos pedidos de inscrição e aos padrões exigidos (Baxter, Callaghan e McAvoy, 2018). A procura pela

qualidade vem geralmente de estudantes envolvidos na tentativa de obter um diploma necessário para a mobilidade social e económica (Sin, Tavares e Amaral, 2016). Por outro lado, o recente declínio no apoio financeiro público ao ensino superior global está a ter um impacto significativo em todos os setores do ensino superior (Ortagus e Yang, 2018) e a pandemia global exacerbou a situação. À medida que o custo global do ensino superior aumenta e que os governos rompem com os seus compromissos (Altbach, Reisberg e Rumbley, 2019), as instituições de ensino superior têm explorado oportunidades e novas formas de aumentar o acesso aos seus cursos, através da aprendizagem digital (Deming, Goldin, Katz e Yuchtman, 2015).

Quer isto dizer que as instituições de ensino superior estão a complementar a sua oferta nos espaços físicos tradicionais, de tijolo e argamassa, aventurando-se numa variedade virtual de modelos educacionais preconizados pela economia digital (Zorn, Haywood e Glachant 2018).

Essa tendência está em linha com a necessidade de mudança do ensino superior em virtude do aumento da responsabilidade financeira que cabe às Instituições do Ensino Superior (IES) e da necessária adoção de critérios de produtividade (Waller, Lemoine, Mense, Garretson e Richardson, 2019), a semelhantes ao tecido empresarial.

Em resumo, pressionadas pela exigência interna e externa da qualidade as universidades estão a reconsiderar as suas missões e os seus objetivos, e a usar a tecnologia e o digital como um andaime de mudança.

\section{As solicitações da globalização ao ensino superior}

A globalização exige que o ensino superior desenvolva uma força de trabalho globalmente competitiva, conduza pesquisas e produza inovações da mais alta qualidade ao menor custo possível (Prakash, 2018). No entanto, hoje o ensino superior enfrenta os seus maiores desafios: incerteza económica, maior responsabilidade perante os seus públicos e tecnologias digitais emergentes que são simultaneamente desafiantes e intimidadoras para os estudantes para os professores (Dennis, 2018).

Por um lado, as tecnologias digitais estão a permitir um maior acesso à educação e a novos mercados para recrutar estudantes, originando maiores oportunidades de receita para as instituições globais de ensino superior (Magdalene e Sirdharan, 2018). Por outro, a esse maior acréscimo de públicos junta-se o desafio da eficácia da ação educativa. Para maximizar a sua eficácia no ambiente global em que as IES se movem, é-lhes essencial manter um forte senso de identidade e de 
resolução; por outro lado, é essencial estarem recetivas a modificações e ajustes e envolver outras pessoas, principalmente estudantes (Everhart e Seymour, 2017).

Precisam ainda, estar preparadas para combinar os talentos em que se especializaram com os desafios imediatos criados pela globalização (Waller, Lemoine, Mense e Richardson, 2019). Entre estes incluem-se os problemas ecológicos, sociais, culturais e económicos que exigem soluções inovadoras e novos conceitos. O ensino superior passou a ter que pensar na sustentabilidade e na resiliência afim de garantir a qualidade (Lemoine e Richardson, 2019). Este dilema ( entre a procura da identidade e o desafio de inovar) também obriga as IES a procurar a excelência e a equidade num clima de competição (Dei, 2019; Kenny, 2017).

Aspecto importante desta procura da qualidade reside na redescoberta dos aspetos pedagógicos e da sua importância na excelência da missão educativa (Kaynardağ, 2019). As IES estão a adaptar-se e a modificar os seus modelos de ensino tradicional ( Lemoine, Jenkins, e Richardson, 2017) para modelos centrados no estudante de que a virtualização do ensino/ aprendizagem se tornou expoente.

Esta confluência de fatores exige que as instituições académicas repensem e reestruturem, o que e como elas ensinam e pesquisam, e como elas se cruzam com os estudantes e a sociedade (Neimann e Stelson, 2019). Por causa da globalização, da internacionalização e da mobilidade associada ao desenvolvimento de uma formação de maior qualidade (Sin, Veiga e Amaral, 2016), os estudantes foram forçados a interagir em ambientes de aprendizagem digital: com o conteúdo, entre os pares e com a instituição. Essa exigência tem vindo a potenciar o desenvolvimento de uma cidadania digital, que é também desejada pelos organismos internacionais. Todavia a questão que emerge é a de saber se essa cidadania se desenvolve e aprofunda por efeito da pandemia, ou, se pelo contrário se limita ao desenvolvimento de algumas competências mais técnicas.

\section{O papel da tecnologia na transformação do ensino superior}

A tecnologia, como parte omnipresente no "mercado" da educação, tornou-se central nas mudanças globais, remodelando a vida social, económica e cultural. A tecnologia não criou o conceito nem a importância da internacionalização que qualquer Universidade inclui na sua missão. No entanto, todas sentiram o efeito do impacto da tecnologia digital na prossecução dessa missão (McKenna, 2018), tanto em termos de educação (instrução / ensino / aprendizagem) quanto de possibilidade de alargamento do universo de recrutamento de estudantes - no ano de 2019/2020 havia 60679 estudantes estrangeiros a frequentar IES Portuguesas - 15, 3\% do total de inscritos - (DGEEC,2020.) 
Numa outra linha argumentativa, a tecnologia veio facilitar os processos de disseminação e de acesso ao conhecimento produzido, assegurando a sua divulgação mais rápida, tornando-se essencial para a criação, intercâmbio e implementação do conhecimento. A partilha do saber e da informação passaram a ser mais valorizados e globalizados do que nunca (Rahim, Burell e Duncan, 2020). Um- efeito dessa importância constata-se nos serviços de apoio tecnológico que aumentam a eficiência das IES, a sua complexidade, e os custos da sua utilização (Waller, Lemoine e Richardson, 2019).

Todavia, o efeito maior da tecnologia fez-se sentir ao nível das possibilidades que abriu para o ensino/ aprendizagem. A maioria das IES fez investimentos significativos em tecnologias digitais educativas, num esforço de melhorar a oferta curricular e as práticas pedagógicas, de diversificar as estratégias didáticas e as formas de comunicação entre docentes e estudantes; de aumentar a participação dos estudantes como agentes da sua aprendizagem (Sursock, 2015; Gaebel e Zhang, 2018).

Apesar dessas possibilidades abertas, ainda se encontra alguma adoção inconsistente da tecnologia educacional em certas IES. Tal ocorre devido às infraestruturas deficitárias, à obsolescência tecnológica, ou à falta de implementação e aplicação das tecnologias digitais (Grant, 2019).

Em suma, o maior desafio para as IES, no que concerne ao uso pedagógico da Tecnologia é o de encontrar as melhores formas de potenciar a preparação e a aprendizagem dos estudantes. Numa apreciação geral e até ao momento, o uso da tecnologia tem sido principalmente evolutivo e não revolucionário (Axtell e Asino, 2020). O desafio é projetar programas curriculares economicamente eficientes, educacionalmente eficazes que atraiam os estudantes e produzam "graduados" competentes e capazes (Padmo, Idrus e Ardiasih, 2019).

\section{A aprendizagem digital no Ensino Superior}

Mesmo tendo em consideração as limitações e problemas referidos no último parágrafo, é possível dizer que a utilização da tecnologia digital no Ensino Superior transformou a maneira como os estudantes aprendem e a maneira como os professores ensinam e usam os aplicativos digitais para ajudar na aprendizagem (Rust, Brinthaupt e Adams, 2017).

A educação no passado costumava ser uma experiência passiva para o estudante, na qual o conhecimento era apresentado, absorvido e o grau de retenção avaliado. Ao invés, para as competências desejadas no século XXI, a aprendizagem precisa de ser mais do que mera retenção, 
requer o desenvolvimento de habilidades que possam ser aplicadas de forma produtiva (Farhan, Talib e Mohammed, 2019; Ketritfhi, Lipshuetz e Santiague, 2017).

A aprendizagem digital potencia essa mudança na medida em que exige uma maior participação do estudante e uma sua corresponsabilidade nos processos pedagógicos que lhe são propostos. Fazendo uma revisão da literatura sobre o assunto, Raby, Karsenti, Meunier e Villeneuve (2011) indicam que a introdução das TDIC na educação universitária traz muitas vantagens para os estudantes, em termos de acesso à informação, de envolvimento, de aprendizagem em profundidade do assunto ou mesmo de facilitação das interações entre o docente e os estudantes (Raby et al., 2011).

Especificamente, tais processos são caracterizados pela diversidade pedagógica, pela flexibilidade das estratégias (Bala, 2018; Chaudhary, Shahzadi e Mahmood, 2019) e das condições de aprendizagem, a que corresponde, do lado do papel do estudante, à sua corresponsabilidade na construção do seu saber.

De facto as ferramentas digitais permitem garantir a continuidade pedagógica e a aprendizagem a qualquer hora e em qualquer lugar, muitas vezes de forma mais significativa e criativa, o que operacionaliza essa corresponsabilidade. Igualmente, porquanto se flexibiliza o tempo para aprender enfatiza-se a sua dimensão longitudinal, o que permite ao estudante ver o caminho percorrido e coadjuvar o processo de monitorização da aprendizagem (Ossiannilsson, 2018). Também a possibilidade da colaboração de pares que as ferramentas digitais permitem, ajuda a enfatizar o envolvimento dos estudantes.

Desse modo, a aprendizagem digital torna-se uma mais valia porque, além de diversificar as abordagens e estratégias educacionais, facilita a aprendizagem, porque dá mais "espaço" ao estudante; permite uma personalização do ensino / aprendizagem; permite a colaboração entre estudantes; desenvolve / aumenta o envolvimento, autonomia e criatividade dos alunos.

Quando se pensa nos efeitos que a adoção da aprendizagem digital acarreta para o trabalho dos professores, constata-se numa primeira "abordagem" que a tarefa de ensinar não é mais a mesma. De acordo com o relatório sobre a estratégia nacional para o ensino superior em França (Béjean e Monthubert, 2015), a aprendizagem digital constituiria uma oportunidade de renovação educacional porque permitiria que o papel do professor se renovasse no panorama educacional. Se o professor já não é o detentor exclusivo do conhecimento, tal significa que lhe cabem agora funções de articulação e projetivas desempenhadas a partir da função mais interativa do ensino. Quer isto dizer que ao docente comete a responsabilidade de fornecer e enquadrar experiências de aprendizagem que estimulem a corresponsabilidade dos estudantes na construção e organização 
do seu conhecimento, que criem espaço para o desenvolvimento da competência reflexiva e autoavaliativa dos estudantes Ao mesmo tempo, a dispensa de ser a fonte única do conhecimento contribui para a libertação do tempo pedagógico (liberation du temps pédagogique) de que falam Dubrac e Djebara (2015), que agora deixa de estar encapsulado em blocos temporais fixos, em que “jorrava o saber”.

Em suma, a aprendizagem digital constitui um desafio ao modo de ensinar dos professores. Espera-se que os docentes centrem a sua ação nos estudantes (agora entendidos como principais agentes do seu processo de aprendizagem) e na escolha de situações de aprendizagem e de avaliação que permitam aos estudantes:

- Aprender com base no conhecimento anterior;

- Explorar as interações com os pares e com o professor;

- Responsabilizar-se pela construção e organização do conhecimento;

- Desenvolver a capacidade reflexiva;

- Corresponsabilizar-se pela sua autoavaliação;

- Contextualizar a aprendizagem em situações reais.

\section{Os desafios da aprendizagem digital em contexto de pandemia}

À primeira vista, poderíamos considerar que a aprendizagem digital se adequa perfeitamente às restrições impostas pela pandemia às e nas IES. Como foi dito na secção anterior, a aprendizagem em ambientes digitais, quando comparada com as formas mais típicas de ensino presencial, exigiu maior responsabilidade ao estudante, mais e melhor organização, exigiu que desenvolvesse mais e melhores competências transversais. Em situação de pandemia e tendo em consideração a circunstância de confinamento de muitos atores educativos, tais exigências tornaram-se ainda mais pertinentes.

De facto, a diversidade de novas populações de estudantes já havia exigido que as instituições desenvolvessem programas para atender a uma ampla gama de requisitos de aprendizagem da maneira mais rápida possível em termos de tempo, espaço e custo (Abernathy e Thornburg, 2020; Orakci, 2020). Com a pandemia, esse esforço tornou-se a regra e sobrecarregou a competência e a capacidade das IES para lhe responder. Retoma-se a ideia que a aprendizagem digital teve um impacto fundamental na estrutura existente do ensino superior (Montebello, 2019).

Se é facto que a aprendizagem digital não tem limitações, precisa de um corpo docente capaz de reconfigurar as práticas pedagógicas, para o que será necessária alguma criatividade 
(Connelly e Miller, 2018; Oluwafemi e Laseinde, 2019). Também ao estudante se exige mais maturidade do que é habitual nas circunstâncias presenciais, que se correlaciona com a maior autonomia do estudante. Esta caraterística está relacionada com algum stress acrescido do estudante e do docente (Duari e Sarkar, 2019). Isso mesmo foi constatado por muitas universidades durante o período da pandemia, porque nem professores nem estudantes estavam preparados para a rápida mudança de local em que a aprendizagem e o ensino acontecem (Sá e Serpa,2020).

Numa linha de raciocínio semelhante à que foi proposta às IES, por efeito do processo de Bolonha (González, e Wagenaar, 2003; Beneitonne et al., 2007) , trata-se de fazer uma mudança de paradigma. Os professores são convidados a ensinar os estudantes a pensar criticamente, mas agora, em vez de orientar os estudantes na construção dos saberes, também devem ajudar os alunos a entender onde e como encontrar conhecimento e informação com a tecnologia (CresswellYeager, Aucoin e Budenz, 2020; Waller, Garretson, Lemoine e Richardson, 2020).

Especificamente o sobre-esforço colocado nos recursos tecnológicos e nos recursos humanos de apoio, os efeitos na preparação dos docentes e no trabalho dos estudantes sistematizam os principais reptos que a pandemia veio colocar às IES.

Faz sentido, por isso, concluir este pequeno texto com um exercício hipotético que identifique os cenários da aprendizagem online após a pandemia.

1. Ninguém sabe realmente, mas há especulações crescentes de que a aprendizagem online será usada mais do que nunca.

Várias suposições apoiam essa conclusão. A primeira diz respeito aos custos económicos. O foco nas finanças deve ser óbvio - governos, estudantes e partes interessadas procurarão a reavaliação de todas as despesas. Alguns governos como por exemplo a Austrália, a Nova Zelândia e alguns estados nos Estados Unidos já reduziram drasticamente os gastos com a redução de pessoal (Ali, 2020).

Nos países europeus as lógicas governativas têm privilegiado o teletrabalho, sempre que possível, bem como a educação a distância (EILO,2017; OECD,2020).

2. A tecnologia emergiu como uma ferramenta educacional estrategicamente crítica, ligada ao uso extensivo de tecnologias síncronas e assíncronas para o ensino e aprendizagem durante a pandemia (Albrahim, 2020). O desafio institucional será determinar o papel da tecnologia, a sua influência e o seu lugar no ensino / aprendizagem. Os administradores das IES também perceberão a necessidade de desenvolvimento e de formação dos professores para a 
implementação bem-sucedida de uma aprendizagem online de qualidade. Como resultado, a aprendizagem online, ou pelo menos o sistema b-learning, provavelmente, serão uma prioridade tática em quase todas as instituições académicas (Tereseviciene, Trepule, Dauksiene, Tamoliune e Costa, 2020).

3. A maioria das universidades irá, certamente, examinar criticamente todos os programas /currículos e determinar aqueles que são capazes de poder ser oferecidos com uma forte componente online e que possam ser atrativos, tanto nas inscrições como na inserção no mercado de trabalho (Alphonso, 2020). Por outro lado também as inscrições em cursos não formais, não graduados poderão aumentar, traduzindo nesse aumento a influência do mercado de trabalho no currículo.

Uma circunstância específica diz respeito à oferta formativa criada pelas IES e destinada aos estudantes que voltam às IES para fazer outras formações ou aprofundar as que já possuem. Anteriormente, os estudantes ingressavam novamente no circuito académico para subir nas carreiras ou para melhorar as suas chances de emprego no mercado de trabalho. A atual crise económica provocada pela pandemia, poderá determinar novas necessidades de formação e desenhar novas geometrias de articulação institucional capazes de ajudar na recuperação económica (Blevins, Bryant, Krishman e Law, 2020).

4. Além disso, os confinamentos, o aumento das taxas de desemprego, conduziu muitas pessoas à procura de formação para poder voltar ao mercado de trabalho. Muitos desses programas poderiam dar resposta se fossem na modalidade de aprendizagem online / ensino a distância (Huang, Liu, Guo, Yang, Zhao, Wei, Knyazeva, Li, Zhuang, Looi e Chang, 2020). Será que as universidades estão preparadas para responder a esses trabalhadores "deslocados" e desempregados?

5. É provável que haja uma queda drástica no número de estudantes internacionais estudando no exterior, à medida que o mundo muda de uma visão globalizada para uma visão mais local ou regional. Eixos fundamentais das IES no enquadramento que o processo de Bolonha lhes conferiu, a mobilidade e a internacionalização dos estudantes e dos docentes, serão drasticamente modificadas pelos efeitos da pandemia. A competição por estudantes internacionais entre as IES provavelmente irá intensificar-se à medida que o número geral de estudantes internacionais em todo o mundo diminuir. A alternativa será criar mais 
oportunidades para atender estudantes internacionais por meio do ensino a distancia (Marginson, 2020). Por outro lado também é possível um aumento da mobilidade virtual de docentes e discentes, nomeadamente pelo seu baixo custo e facilidades logísticas.

6. As questões pedagógicas associadas à aprendizagem digital adquiriram uma renovada importância com a pandemia. A tecnologia, que pode ser útil para permitir algumas formas de interação entre professores e estudantes e estudantes-estudantes, mas o aspecto crucial de uma aprendizagem eficaz é uma experiência de uma aprendizagem bem delineada e de um professor competente (Jones e Sharma, 2020). Por isso, uma reflexão sobre o seus usos são essenciais no ecossistema de aprendizagem. Temas como os que seguem irão constituir aspetos importantes das práticas que sobreviverão à pandemia:

- A diversidade das pedagogias passa a ser um recurso que deve ser partilhado.

- Tempo, ritmo e espaço precisam de ser reinventados.

- A incerteza e o desconhecido tornam-se temas de aprendizagem.

- A emergência leva ao desenvolvimento de comunidades interdisciplinares.

- Torna-se necessário repensar as formas e modelos de avaliação.

Em suma, à pergunta geral sobre o que é que as IES aprenderam sobre aprendizagem digital durante a pandemia, e como a reconfigurarão após o surto, importa sublinhar o desafio ainda em aberto que é o de não deixar de fora os estudantes a quem a pandemia aumentou as vulnerabilidades, nem deixar de fora o apoio que os professores precisam para fazer da aprendizagem digital uma verdadeira oportunidade ( Sá e Serpa, 2020).

\section{Referências}

ABERNATHY, D. F.; THORNBURG, A. W. Theory and application in the design and delivery of engaging online courses: Four key principles that drive student and instructor engagement and success. In: Handbook of research on developing engaging online courses. Hershey, PA: IGI Global, p.246258, 2020.

ALBRAHIM, F. A. Online teaching skills and competencies. Turkish Online Journal of Educational Technology, 19(1),2020.

ALI, W. Online and remote learning in higher education institutes: A necessity in light of COVID-19 pandemic. Higher Education, 10(3), 2020. 
ALPHONSO, C. The educational world has been turned upside down-- Online learning may reshape the classroom. The Globe and Mail. 2020. Disponível

https://www.theglobeandmail.com/canada/article-as-online-learning-rolls-out-education-maychange-forever/.

ALTBACH, P. G.; REISBERG, L.; RUMBLEY, L. E. Trends in global higher education: Tracking an academic revolution. Leiden, Netherlands: BRILL, 2019.

AXTELL, S.; ASINO, T. I. Emerging information technology issues in higher education. In Makewa,L.N. ; Ngussa,B.M. (eds.) IT issues in higher education: Emerging research and opportunities. Hershey, PA: IGI Global, p.1-16, 2020.

AMARAL, A. ; TAVARES O.; Bases da organização do sistema: diversidade, acesso e equidade In Rodrigues,M.L. ; Heitor,M. (org.) 40 anos de politicas de Ciência e de Ensino Superior, Coimbra, Almedina, p. 539-557, 2015.

BALA, M. Use of ICT in higher education. In: Multidisciplinary Higher Education, Research, Dynamics \& Concepts: Opportunities \& Challenges for Sustainable Development, 1(1), p. 368-376, 2018.

BAXTER, J., CALLAGHAN, G.; MCAVOY, J. Introduction to chapters: Creativity and critique in online teaching and learning: Innovations in online pedagogy. In: Creativity and critique in online learning . Cham, Switzerland: Palgrave Macmillan, p.1-12, 2018.

BÉJEAN, S. Et.; MONTHUBERT, B. Pour une société apprenante - propositions pour une stratégie nationale de l'enseignement supérieur. In: Rapport au président de la république. Paris: Ministre de l'Éducation nationale, de l'Enseignement supérieur et de la Recherche, 2015.

BENEITONE, P; ESQUETINI, C.; GONZÁLEZ, J.; MARTY MALETA, M.; SIUFI, G. ; WAGENAAR, R. Reflections and perspectives of Higher Education in Latin America. In: Final Report, Tuning Latin America Project 2004-2007. Spain, University of Deusto Publications, 2007.

BLEVINS, F.; BRYANT, J.; KRISHNAN, C.; LAW, J. Coronavirus: How should US higher education plan for an uncertain future. Washington, DC: McKinsey and Company, 2020.

CHAUDHARY, F. R.; SHAHZADI, I.; MAHMOOD, A. Comparative study of satisfaction and problems of face to face and online mode of learners. In: International Journal of Distance Education and E-Learning, 4(1), 2019.

CONNELLY, J. O.; MILLER, P. Improving learning outcomes for higher education through smart technology. In: International Journal of Conceptual Structures and Smart Applications (IJCSSA), 6(1), p.1-17, 2018.

CRESSWELL-YEAGER, T. J.; AUCOIN, J.; BUDENZ, D.. A framework for student engagement: Strategies for faculty teaching online. In: Handbook of research on creating meaningful experiences in online courses. Hershey, PA: IGI Global, p. 153-171, 2020.

DEI, D. G. J.; Assessing quality assurance practices in institutions of higher learnings. International Journal of Learning, Teaching and Educational Research, 18(12), p. 30-45, 2019. 
DEMING, D. J.; GOLDIN, C.; KATZ, L. F.; YUCHTMAN, N. Can online learning bend the higher education cost curve? In: American Economic Review, 105(5), p.496-501, 2015.

DENNIS, M. J. The impact of technology on US and worldwide higher education.In Enrollment Management Report, 21(10), p.1-3, 2018.

DGEEC. Principais resultados do Inquérito ao Registo de Alunos Inscritos e Diplomados do Ensino Superior. In RAIDES 19. DGEEC: Lisboa, 2020.

DUBRAC, D ET DJEBARA, A. La pédagogie numérique : un défi pour l'enseignement supérieur. Avis. Paris: Conseil économique, social et environnemental, 2015.

EUROFOUND AND THE INTERNATIONAL LABOUR OFFICE. Working anytime, anywhere: The effects on the world of work, Publications Office of the European Union, Luxembourg, and the International Labour Office, Geneva, 2017.

ENGLUND, C.; OLOFSSON, A. D.; PRICE, L. Teaching with technology in higher education: Understanding conceptual change and development in practice. In: Higher Education Research \& Development, 36(1), p.73-87, 2017.

EVERHART, D.; SEYMOUR, D. M. Challenges and opportunities in the currency of higher education. In: Handbook of research on competency-based education in university settings. Hershey, PA: IGI Global, p. 41-65, 2017.

FARHAN, M. K.; TALIB, H. A.; MOHAMMED, M. S. Key factors for defining the conceptual framework for quality assurance in e-learning. In: Journal of Information Technology Management, 11(3), p. 16-28, 2019.

GAEBEL, M. ; ZHANG T. Learning and teaching in the European Higher Education Area. Berlin: European University Association, 2018.

GONZÁLEZ, J. ; WAGENAAR, R. Tuning Educational Structures in Europe. Final Report - Pilot Project Phase 1. Spain, University of Deusto Publications, 2003.

GRANT, M. M. Difficulties in defining mobile learning: Analysis, design characteristics, and implications. Educational Technology Research and Development, 67(2), p. 361-388, 2019.

HUANG, R. H.; LIU, D. J.; GUO, J.; YANG, J. F.; ZHAO, J. H.; WEI, X. F.; KNYAZEVA, S.; LI, M.; ZHUANG, R. X.; LOOI, C. K.; CHANG, T. W. Guidance on flexible learning during campus closures: Ensuring course quality of higher education. In: COVID-19 outbreak. Beijing: Smart Learning Institute of Beijing Normal University, 2020.

JONES, K.; SHARMA, R. S. Does online learning provide a 'real' college education?, 2020.

KAYNARDAĞ, A. Pedagogy in HE: does it matter? In: Studies in Higher Education, 44:1, p.111119, 2019. DOI: 10.1080/03075079.2017.1340444

KEBRITCHI, M.; LIPSHUETZ, A.; SANTIAGUE, L. Issues and challenges for teaching successful online courses in higher education: A literature review. In: Journal of Educational Technology Systems, 46(1), p. 4-29, 2017.

KENNY, J. Academic work and performativity. In: Higher Education, 74(5), p. 897-913, 2017. 
LEMOINE, P. A.; RICHARDSON, M. D. Creative disruption in higher education: Society, technology and globalization. In Peres,P.; F. Moreira, F.; A. Mesquita,A. (eds). Educational and social dimensions of digital transformation in organizations. Hershey, PA: IGI Global, p. 275-293, 2019.

LODGE, J. M., ; BONSANQUET, A. Evaluating quality learning in higher education: Reexamining the evidence. In: Quality in Higher Education, 20(1), p. 3-23, 2014.

MAGDALENE, R.; SRIDHARAN, D. Powering e-learning through technology: An overview of recent trends in educational technologies. In: The Online Journal of Distance Education and eLearning, 6(1), p.60, 2018.

MARGINSON, S. Global HE as we know it has forever changed. In: Times Higher Education, 2020.

MCKENNA, K. Technology policies and practices in higher education. In: M. Khosrow-Pour (ed). Encyclopedia of information science and technology, Fourth Edition Hershey, PA: IGI Global, p. 39543962, 2018.

MONTEBELLO, M. (ed.). Handbook of research on digital learning. Hershey, PA: IGI Global, 2019.

NEIMANN, T. D.; STELSON, U. M. (eds.). Challenges and opportunities in global approaches to education. Hershey, PA: IGI Global, 2019.

OECD The Territorial Impact Of Covid-19: Managing The Crisis Across Levels Of Government. Paris: OECD 2020.

OLUWAFEMI, I.; LASEINDE, T. Perception of quality in higher education institutions: A logical view from the literature. In: International conference on buman systems engineering and design: Future trends and applications . Cham, Switzerland: Springer, p. 1075-1083, 2019.

ORAKC1, Ş. The future of online learning and teaching in higher education. In: A. M.A. Musleh Al-Sartawi, K. Hussainey A. Hannoon, \& A. Hamdan (Eds.). Global approaches to sustainability through learning and education. Hershey, PA: IGI Global, p. 28-45, 2020.

ORTAGUS, J. C.; YANG, L. An examination of the influence of decreases in state appropriations on online enrollment at public universities. In:Research in Higher Education, 59(7), p. $847-865,2018$.

OSSIANNILSSON, E. Promoting active and meaningful learning for digital learners. In J. Keengwe (ed). Handbook of research on mobile technology, constructivism, and meaningful learning. Hershey, PA: IGI Global, p. 294-315,2018.

PADMO, D.; IDRUS, O.; ARDIASIH, L. S. The utilization of mobile devices for improving access to online learning for distance education's students. In: Turkish Online Journal of Distance Education, 20(2), p. 147-161, 2019.

PRAKASH, G. Quality in higher education institutions: Insights from the literature. In The TQM Journal, 30(6), p. 732-748, 2018. 
RABY, C.; KARSENTI, T.; MEUNIER, H.; ET VILLENEUVE, S. Usage des TIC en pédagogie universitaire : point de vue des étudiants. In: Revue internationale des technologies en pédagogie universitaire, 8(3), p. 6-19, 2011.

RAHIM, E.; BURRELL, D. N.; DUNCAN, T. Best practices and emerging trends for knowledge-based organizations and academic institutions around elearning. In: International Journal of Smart Education and Urban Society (IJSEUS), 11(2), p.16-27, 2020.

RUST, D. Z.; BRINTHAUPT, T. M.; ADAMS, C. L. Using technology to enhance student and faculty success in online courses. In P. Tripathi, S. Mukerji (eds.) Handbook of research on technologycentric strategies for higher education administration. Hershey, PA: IGI Global, p. 195-209, 2017.

SÁ, M. J.; SERPA,S. The Covid-19 Pandemic As An Opportunity To Foster The Sustainable Development Of Teaching In: Higher Education?. Sustainability, 12, no. 20. p. 1-16, 2020.

SIN, C. ; AMARAL;,A. V. Academics' And Employers' Perceptions About Responsibilities For Employability And Their Initiatives Towards Its Development?. In: Higher Education 73, no. 1, p.97-111, 2017.

SIN, C.; AMARAL.A. V. European Policy Implementation And Higher Education. Analysing The Bologna Process. London: Palgrave Macmillan, 2016.

SIN, C.; TAVARES, O.; AMARAL, A. Who is responsible for employability? Student perceptions and practices. In: Tert Educ Manag, 22, p. 65-81, 2016 Disponível https://doi.org/10.1080/13583883.2015.1134634.

SURSOK, A. Trends 2015 earning and Teaching in European Universities. Brussels. European Universities Association, 2015.

TERESEVICIENE, M.; TREPULE, E.; DAUKSIENE, E.; TAMOLIUNE, G.;COSTA, N. Are universities ready to recognize open online learning? In: International Education Studies, 13(2), p.2132, 2020.

VAVRUS, F. ET AL; BARTLETT. Comparative Pedagogies and Epistemological Diversity : Social and Materials Contexts of Teaching. In: Tanzania Comparative Education Review, 56 (4), p. 634-655. 2012.

WALLER, R. E.; LEMOINE, P. A.; MENSE, E. G.; RICHARDSON, M. D.Higher education in search of competitive advantage: Globalization, technology and e-learning. In: International Journal of Advanced Research and Publications, 3(8), p.184-190, 2019.

ZORN, A.; HAYWOOD, J.; GLACHANT, J.M. (eds.) Higher education in the digital age. Moving academia online. Cheltenham, UK: Edward Elgar Publishing Limited, 2018.

Recebido em: 18 nov. 2020/ Aprovado em: 01 dez. 2020

Cite como

(ABNT NBR 6023:2018) 
NOBRE, Ana; MOURAZ, Ana. Reflexões sobre os efeitos da pandemia na aprendizagem digital. Dialogia, São Paulo, n. 36, p. 367-381, set./dez. 2020. Disponível em: https://doi.org/10.5585/dialogia.n36.18722.

\section{American Psychological Association (APA)}

Nobre, A., \& Mouraz, A. (2020, set./dez.). Reflexões sobre os efeitos da pandemia na aprendizagem digital. Dialogia, São Paulo, 36, p. 367-381. https://doi.org/10.5585/dialogia.n36.18722. 